\title{
Potentiation of the amnesic effect of electroconvulsive shock by detention'
}

\author{
FRANCESCO ROBUSTELLI, ANNE GELLER AND MURRAY \\ E. JARVIK, ALBERT EINSTEIN COLLEGE OF MEDICINE. \\ Bronx, N.Y. 10461
}

Electroconvulsive shock (ECS) administered to mice $10 \mathrm{~min}$ after training in a one-trial passive avoidance situation decreases their step-through latencies in the retest trial given $24 \mathrm{~h}$ later. $A$ similar degree of impairment is found if the animals are detained in the same compartment of the conditioning apparatus for 10 min immediately after the training trial. If ECS is given at the end of the detention period, its amnesic effect on the conditioned response is greatly increased.

In a previous paper Robustelli \& Jarvik (1968) have reported: (1) mere detention of mice in the safe compartment of a passive avoidance conditioning apparatus after training can produce a decrease in the step-through latencies in the retest trial given $24 \mathrm{~h}$ later; (2) this decrease of retest latencies is produced only if the detention in the safe compartment occurs immediately after the training trial, but seems to be absent if the detention occurs 12 or $23 \mathrm{~h}$ later; (3) detention in the safe compartment can potentiate the amnesic effect of an ECS given immediately after the end of the detention period; (4) the decrease of retest latencies can be obtained by detaining the animals not only in the safe compartment of the apparatus, but also in the compartment in which the shock was administered and even in a jar outside the apparatus. In regard to finding (3), it was assumed that detention and ECS both act in the same direction, interfering with retention of the passive avoidance response and that their effects are summed in Ss receiving both treatments. The present investigation was designed to test this assumption using a shipment of animals which were shown to be less sensitive to the effects either of detention or ECS than the shipment used by Robustelli and Jarvik in the previous experiments.

Subjects

The Ss were 160 female CFI albino mice approximately six weeks old, housed 8 to a cage, with ad lib access to food and water.

Apparatus and Procedure

The step-through apparatus consisting of a trough-shaped two-compartment box as described by Jarvik \& Kopp (1967) was used. In the smaller transparent compartment Ss were exposed to a bright Tensor lamp approximately $2 \mathrm{~cm}$ away. The smaller compartment was connected with the larger darkened compartment by an opening that could be closed by a guillotine door. The Ss were placed into the smaller lit compartment facing away from the door and they received a punishing shock $(350 \mu \mathrm{A})$ for stepping spontaneously into the larger darkened compartment. The Ss escaped from the foot shock back into the lit compartment, after which the guillotine door was closed preventing reentry into the shock compartment. The step-through latencies were redetermined $24 \mathrm{~h}$ after the learning trial. Animals tarrying longer than $300 \mathrm{sec}$ were removed. Electroconvulsive shock (15 $\mathrm{mA}$ for $0.2 \mathrm{sec}$ ) producing full tonic seizures, was administered transcorneally using a Hans electroshock apparatus (2-C).

Four groups of animals were used with 40 animals in each group. The Ss of Group A were returned to their home cages immediately after the training trial and given a sham ECS $10 \mathrm{~min}$ later. The $S$ s of Group B were detained immediately after the training trial for $10 \mathrm{~min}$ in the smaller lit compartment of the apparatus. They were removed and given sham ECS before being returned to their home cages. The $S$ s of Group $C$ were returned to their home cages immediately after the training trial and given ECS treatment $10 \mathrm{~min}$ later. The Ss of Group D were detained for $10 \mathrm{~min}$, removed, and then were given ECS before being returned to their home cages.

\section{Results}

The results of the retest trials are represented in Fig. 1. As can be seen, in Groups B and C, in which detention only, and ECS only, were given after the training trial, latencies are very similar and slightly, though significantly lower $(p<$ than 0.02 ) (Wilcoxon $\&$ Wilcox, 1964) than in the control group. On the contrary, in Group D, in which ECS was administered at the end of the detention period, there is a remarkable reduction of latencies in the retest trials. The difference between the scores of this group and the scores of all other three groups is highly significant $(\mathrm{p}<0.01)$.

\section{Discussion}

These results confirm in a striking way the previous finding of a potentiation of the ECS effect by detention. The relatively slight decrease in retest latencies found in the group given only ECS is in strong contrast with the enormous impairment of performances in the group which has been given ECS preceded by a detention period.

Recently, Davis \& Agranoff (1966) have found that detaining goldfish immediately after the training session in the apparatus in which it had been previously conditioned appeared to prolong the presumed consolidation process. In fact, puromycin administered intracranially at the end of the detention period produced a retrograde amnesia whereas its administration at the same time after training to animals who had not been detained was without effect. It was concluded that detention prolonged the puromycin susceptible phase of memory consolidation. However, they could not show any amnesic effect of detention when given alone.

Subsequently, Davis (1968) has reported that mere detention of goldfish for very long periods ( 24 or $48 \mathrm{~h}$ ) can produce retrograde amnesia. But at this time it was not possible to detect any potentiation of the puromycin effect with such long periods of detention. Thus, in these two papers, no potentiation of the puromycin effect has been shown with detention, when detention was effective by itself. However, the many differences between the experiments of Davis and Agranoff and ours, like amnesic treatment (puromycin versus ECS), animal species (goldfish versus mouse), conditioning technique (mulitple-trial shuttle-box versus one-trial passive avoidance), can account for the different results.

In the previously cited paper Robustelli \& Jarvik (1968) suggested that detention could be a kind of generalized extinction

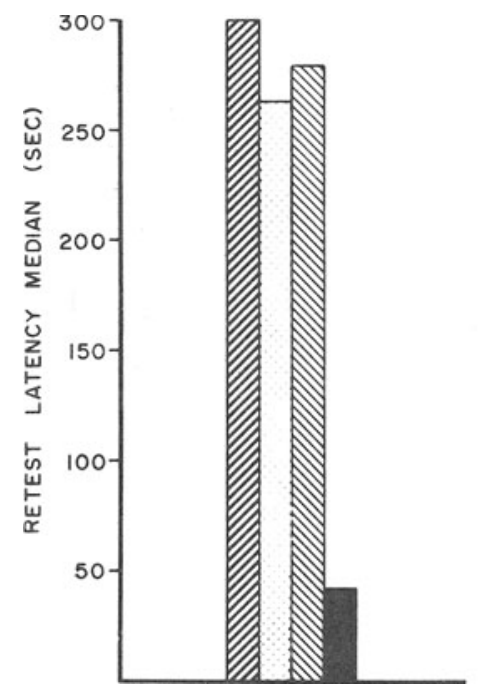

CONTROL

DETENTION

ECS

DETENTION \& ECS

Fig. 1. Median retest latencies of mice $24 \mathrm{~h}$ after training. 
or, less specifically, a new experiential event that can retroactively interfere with the previous learning experience. This hypothesis, which still seems to be the most plausible on the basis of the experiments described in that paper, raises serious difficulties regarding the assumption of a selective amnesic effect of ECS on the most recent learning experience, as has been previously proposed (Bures \& Buresova, 1963; Duncan, 1948; Gerbrandt \& Thomson, 1964; Hamilton \& Patton, 1952). In fact, in these studies ECS administered after extinction or reversal resulted in an improvement of the original learning performances of the animals, compared with the performances of the animals which had been given extinction or reversal trials only. Whatever detention is, under our experimental conditions it has been shown to be an amnesia producing experience. Thus, since ECS is administered at the end of the detention period, it would be reasonable to expect that it would affect the detention experience more than the original conditioning, the detention being closer to the ECS in time. One would predict that, because of the selective effect on the detention, amnesia for the original conditioning should be less, or even absent, when both treatments are administered together. It could, however, be objected that the particular technique and the particular procedure we have used do not justify a prediction on the basis of a selective effect of ECS upon the experience more closely preceding its administration. That is, since we have used a single training trial, which on the average lasts for about $20 \mathrm{sec}$, and the detention period is $10 \mathrm{~min}$, the whole complex of the training and extinction procedure has a duration of no more than 11 min. Thus, it would be reasonable to expect that the ECS administration affects the whole complex of both experiences rather than having a selective action on the extinction experience, because of the immediate temporal sequence of training and extinction. But, even if this were so, ECS given after the detention period should have at most the same effect as ECS given alone and the strong potentiating effect of detention on ECS remains unexplained. As a matter of fact, at the present stage of our knowledge of the mechanism of action of both ECS and extinction, and also of the possible existence of a consolidation process of the mnemonic trace, it is difficult to say how things would really look if detention were a kind of extinction. What is particularly missing is a theoretical scheme for the possible relationship between extinction and consolidation of a conditioned response.
The hypothesis of Davis and Agranoff, according to which detention might postpone the consolidation of the mnemonic trace, could also be used for our experiment. Still another explanation can be considered. We can suppose that potentiation of ECS is not a consequence of some kind of summation of the amnesic effects of the two treatments, but it is simply a result of an altered physiological state which makes the animal more sensitive to ECS at the end of the detention period. However, the detention phenomenon, whatever are its causes and mode of action, may be considered as a new tool to check and clarify some of the interpretations so far proposed for the action of ECS. Obviously, further investigations are needed, directed towards a systematic analysis of both detention by itself and its interaction with ECS, to have a better idea of the contribution that this new approach can give to the study of the amnesic effect of ECS and to the understanding of memory processes in general.

\section{REFERENCES}

BURES, J., \& BURESOVA, O. cortical spreading depression as a memory disturbing factor. J. comp. physiol. Psychol, 1963, 56, 268-272.

DAVIS, R. E. Environmental control of memory fixation in goldfish. $J$. comp. physiol Psychol, 1968, 65, 72-78.

DAVIS, R. E., \& AGRANOFF, B. W. Stages of memory formation in goldfish: evidence for an environmental trigger. Proc. Nat. Acad. Sci, $1966,55,555-559$.

DUNCAN, C. P. Habit reversal induced by electroshock in the rat. J. comp. physiol Psychol, 1948, 41, 11-16.

GERBRANDT, L. K., \& THOMSON, C. W. Competing response and amnesic effects of electroconvulsive shock under extinction and incentive shifts. $J$. comp. physiol. Psychol., 1964, 58, 208-211.

HAMILTON, C. L., \& PATTON, R. A. The effects of a series of electroshock convulsions on an inhibited conditioned response in the albino rat. $J$. comp. physiol Psychol., 1952, 45, 600-603.

JARVIK, M. E., \& KOPP, R. An improved one-trial passive avoidance learning situation. Psychol. Rep., 1967, 21, 221-224.

ROBUSTELLI, F., \& JARVIK, M. E. Retrograde amnesia from detention. Physiol. Behav., 1968, 3, in press.

WILCOXON, F., \& WILCOX, R. A. Some rapid approximate statistical procedures. Lederle Laboratories, Pearl River, N. Y. 1964.

NOTE

1. Supported by Public Health Service Grant MH 05319. 